\title{
A NOTE ON CYCLES IN LOCALLY HAMILTONIAN AND LOCALLY HAMILTON-CONNECTED GRAPHS
}

\author{
LONG TANG \\ College of Mathematics and System Sciences, \\ Xinjiang University \\ Urumqi 830046, P.R. China \\ e-mail: 1048794282@qq.com \\ AND \\ ELKIN VUMAR ${ }^{1}$ \\ College of Mathematics and System Sciences, \\ Xinjiang University \\ Urumqi 830046, P.R. China \\ e-mail: elkin1226@sina.com
}

\begin{abstract}
Let $\mathcal{P}$ be a property of a graph. A graph $G$ is said to be locally $\mathcal{P}$, if the subgraph induced by the open neighbourhood of every vertex in $G$ has property $\mathcal{P}$. Ryjáček conjectures that every connected, locally connected graph is weakly pancyclic. Motivated by the above conjecture, van Aardt et al. [S.A.van Aardt, M. Frick, O.R. Oellermann and J.P.de Wet, Global cycle properties in locally connected, locally traceable and locally Hamiltonian graphs, Discrete Appl. Math. 205 (2016) 171-179] investigated the global cycle structures in connected, locally traceable/Hamiltonian graphs. Among other results, they proved that a connected, locally Hamiltonian graph $G$ with maximum degree at least $|V(G)|-5$ is weakly pancyclic. In this note, we improve this result by showing that such a graph with maximum degree at least $|V(G)|-6$ is weakly pancyclic. Furthermore, we show that a connected, locally Hamilton-connected graph with maximum degree at most 7 is fully cycle extendable.
\end{abstract}

Keywords: locally connected, locally Hamiltonian, locally Hamilton-connected, fully cycle extendability, weakly pancyclicity.

2010 Mathematics Subject Classification: 05C38; 05 C45.

\footnotetext{
${ }^{1}$ Corresponding author.
} 


\section{REFERENCES}

[1] A. Adamaszek, M. Adamaszek, M. Mnich and J.M. Schmidt, Lower bounds for locally highly connected graphs, Graphs Combin. 32 (2016) 1641-1650. doi:10.1007/s00373-016-1686-y

[2] J.A. Bondy and U.S.R. Murty, Graph Theory (Springer, New York, 2008).

[3] A. Borchert, S. Nicol and O.R. Oellermann, Global cycle properties of locally isometric graphs, Discrete Appl. Math. 205 (2016) 16-26. doi:10.1016/j.dam.2016.01.026

[4] C. Brause, D. Rautenbach and I. Schiermeyer, Local connectivity, local degree conditions, some forbidden induced subgraphs, and cycle extendability, Discrete Math. 340 (2017) 596-606. doi:10.1016/j.disc.2016.11.035

[5] G. Chartrand and R.E. Pippert, Locally connected graphs, Časopis Pěst. Mat. 99 (1974) 158-163.

[6] J.P. de Wet and S.A. van Aardt, Traceablity of locally Hamiltonian and locally traceable graphs, Discrete Math. Theor. Comput. Sci. 17 (2016) 245-262.

[7] J.P. de Wet, M. Frick and S.A. van Aardt, Hamiltonicity of locally Hamiltonian and locally traceable graphs, Discrete Appl. Math. 236 (2018) 137-152. doi:10.1016/j.dam.2017.10.030

[8] G.R.T. Hendry, Extending cycles in graphs, Discrete Math. 85 (1990) 59-72. doi:10.1016/0012-365X(90)90163-C

[9] D.J. Oberly and D.P. Sumner, Every connected, locally connected nontrivial graph with no induced claw is Hamiltonian, J. Graph Theory 3 (1979) 351-356. doi:10.1002/jgt.3190030405

[10] C.M. Pareek, On the maximum degree of locally Hamiltonian non-Hamiltonian graphs, Util. Math. 23 (1983) 103-120.

[11] C.M. Pareek and Z. Skupień, On the smallest non-Hamiltonian locally Hamiltonian graph, J. Univ. Kuwait Sci. 10 (1983) 9-16.

[12] Z. Skupień, On the locally Hamiltonian graphs and Kuratowski's theorem, Bull. Acad. Pol. Sci., Ser. Sci. Math. Astron. Phys. 13 (1965) 615-619.

[13] Z. Skupień, Locally Hamiltonian and planar graphs, Fund. Math. 58 (1966) 193-200. doi:10.4064/fm-58-2-193-200

[14] S.A. van Aardt, M. Frick, O.R. Oellermann and J.P. de Wet, Global cycle properties in locally connected, locally traceable and locally Hamiltonian graphs, Discrete Appl. Math. 205 (2016) 171-179.

doi:10.1016/j.dam.2015.09.022

[15] D.B. West, Research problems, Discrete Math. 272 (2003) 301-306. doi:10.1016/S0012-365X(03)00207-3

Received 22 June 2017

Revised 5 February 2018 Accepted 5 February 2018 